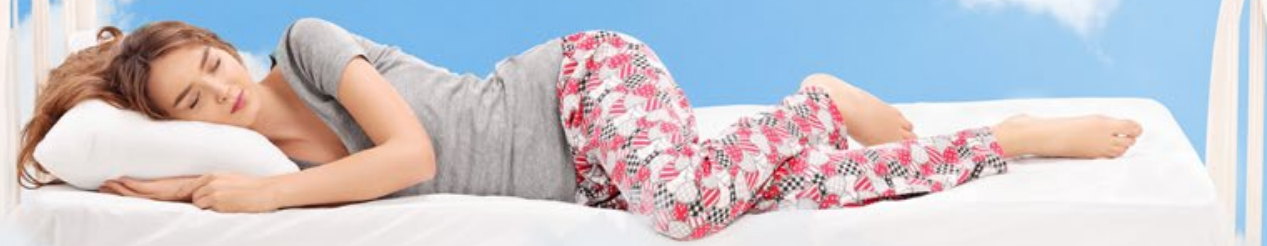

Aufklärung, Verhaltenstherapie, Antidepressiva \& Co.

\title{
Was tun, wenn Schmerzen den Schlaf stören?
}

Schlafstörungen sind bei Schmerzpatienten eher die Regel als die Ausnahme. Manchmal ist eine mangelhafte Medikation die Ursache. Aber auch ein Restless Legs Syndrom (RLS) und eine schlechte Schlafhygiene halten die Patienten nachts häufig wach.

Bei Patienten mit chronischen Schmerzen sind Schlafstörungen häufig: Je nach Untersuchung und Definition klagen die Hälfte bis vier Fünftel der Schmerzpatienten über einen schlechten Schlaf, hat Dr. Peter Geisler vom Schlafmedizinischen Zentrum der Universität Regensburg berichtet. Die Ursachen liegen auf der Hand: „Der Schmerz ist stärker als der Schlaf“, so Geisler auf der Tagung der Deutschen Gesellschaft für Schlafforschung und Schlafmedizin (DGSM) in Mainz. Daher sei es falsch, Schmerzmittel nachts zu reduzieren, weil die Patienten ja schliefen. Denn das geschehe nicht, wenn die Medikamente zu niedrig dosiert würden, so der Schlafmediziner. Wenn, dann sollte eher tagsüber eine Reduktion erfolgen - dann sind die Patienten stärker abgelenkt.

Auf der anderen Seite scheint schlechter Schlaf Schmerzen zu verstärken: „Er ist ein Prädiktor für die Exazerbation von chronischen Schmerzen“, so Geisler.

\section{Genug Schmerzmittel in der Nacht?} Wenn Schmerzpatienten über Schlaflosigkeit klagen, sollten Ärzte zunächst mögliche Ursachen suchen. Ganz oben steht eine ausreichende Schmerzmedikation in der Nacht. Auch die Therapie der Patienten sollte geprüft werden. „Gehen Sie kritisch die Medikamentenliste durch. Was könnte verantwortlich sein für schlechten Schlaf oder Alpträume?" Viele Patienten erhalten Kortikoide, die den Schlaf beeinträchtigen können. Bei Opioiden, so Geisler, ist die zentrale Apnoe zu beachten, und viele andere Medikamente hätten ebenfalls schlafstörende Nebenwirkungen.

\section{Klären Sie Ihre Patienten umfassend auf!}

Schmerzpatienten sind zudem sehr anfällig für Ängste und Depressionen - auch damit könnten die Probleme zusammenhängen, vor allem wenn eine schwere Grunderkrankung vorliegt. Hier sei es wichtig, die Patienten über ihre Erkrankung, die Prognose und die Behandlung gut aufzuklären, um Ängste und Sorgen zu nehmen. Bei terminalen Erkrankungen sollte eine gute Anxiolyse erfolgen.

Jeder zweite Patient mit chronischen Schmerzen im Bewegungsapparat habe auch ein RLS. Das Problem: „Viele Erkrankte erkennen nicht, dass es sich um zwei Arten von Schmerzen handelt: den RLS-Schmerz und den der Grunderkrankung. Hier muss man herausfinden: Was ist das für eine Art von Schmerz, wann tritt er auf und wodurch wird er besser?"

Schließlich, so Geisler, sollten Ärzte auch ungünstiges Verhalten identifizieren. So sind Schmerzpatienten oft kaum in der Lage, sich viel zu bewegen. Auch halten sie sich oft drinnen auf, wo das Licht nicht reicht, um den Tag-Wach-Rhythmus zu synchronisieren. Wenn sie dann noch tagsüber einnicken oder das Bett aufsuchen, sei es kein Wunder, dass sie nachts wachliegen. In Kliniken sei auch darauf zu achten, dass die Pflegetätigkeiten nicht um vier Uhr morgens beginnen. Werden solche Faktoren berücksichtigt und ist den- noch eine Behandlung nötig, so setzt Geisler zunächst auf verhaltenstherapeutische Maßnahmen. Die meisten Elemente einer insomniespezifischen kognitiven Verhaltenstherapie ließen sich auch bei Schmerzpatienten anwenden. Dazu zählt Geisler etwa den Versuch, die Angst vor der Schlaflosigkeit zu mindern, Lichtexposition, Abendrituale oder begrenzte Bettzeiten. Bei Fibromyalgiepatienten habe die Verhaltenstherapie allerdings nur eine geringe Wirksamkeit.

\section{Sedierende Antidepressiva, niedrig dosiert}

Ist eine medikamentöse Therapie erforderlich, könnte ein Versuch mit sedierenden Antidepressiva wie Doxepin und Mirtazapin erfolgreich sein. Oft genügen $<5 \mathrm{mg}$ um den Schlaf anzustoßen. Werden Hypnotika verwendet, seien vor allem $Z$-Substanzen oder kurz wirksame Benzodiazepine wie Brotizolam, Oxazepam, Temazepam oder Triazolam geeignet. Langwirksame Vertreter (Bromazepam, Diazepam oder Lormetazepam) könnten die Schmerzen hingegen verstärken. Chloralhydrat und Antihistaminika hält Geisler ebenfalls für wenig geeignet.

Gerade bei Patienten mit neuropathischen Schmerzen ist Pregabalin eine interessante Option. Das Schmerzmittel hat guten schlaffördernden Effekt, hier müsse möglicherweise nur die Hauptdosis am Abend gegeben werden, um von der Wirkung zu profitieren. Generell sollten Ärzte hiermit aber aufgrund des Abhängigkeitspotenzials vorsichtig umgehen. Die Entzugserscheinungen seien oft schlimmer als die bei einer Benzodiazepinabhängigkeit, so der Schlafmediziner.

(Thomas Müller) 